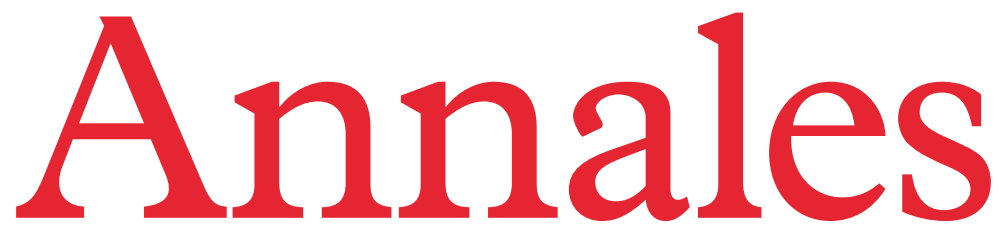

Histoire, Sciences Sociales

\title{
Histoire quantitative
}

Karine Karila-Cohen

Isabelle Rosé

Clare H. Crowston · Steven L. Kaplan · Claire Lemercier

Elsa Génard · Melchior Simioni

Pierre Mercklé · Claire Zalc

\section{Histoire sociale}

$73^{\mathrm{e}}$ année $-\mathrm{n}^{\circ} 4$ octobre-décembre 2018

ÉDITIONS DE L'ÉCOLE

DES HAUTES ÉTUDES

EN SCIENCES SOCIALES

CAMBRIDGE

UNIVERSITY PRESS 


\section{Annales \\ Histoire, Sciences Sociales}

$73^{\mathrm{e}}$ année
$\mathrm{n}^{\circ} 4$ octobre-décembre 2018

\section{Histoire quantitative}

Karine Karila-Cohen, Claire Lemercier,

Isabelle Rosé et Claire ZALC

Nouvelles cuisines de l'histoire quantitative

Karine Karila-COHEN

Le graphe, la trace et les fragments

L'apport des méthodes quantitatives et des outils numériques à l'étude des élites civiques athéniennes

Isabelle Rosé

Autour de la reine Emma (vers 890-934)

Réseaux, itinéraire biographique féminin et questions documentaires au début du Moyen Âge central

Clare H. Crowston, Steven L. Kaplan et Glaire Lemercier

Les apprentissages parisiens aux XVIII ${ }^{\mathrm{e}}$ et XIX ${ }^{\mathrm{e}}$ siècles

Elsa GÉnARD et Melchior SimIONI

Une histoire politique des chiffres de la prison

Conception, production et usages de la Statistique pénitentiaire (1852-1939)

Pierre Mercklé et Claire Zalc

Peut-on modéliser la persécution?

Apports et limites des approches quantifiées sur le terrain de la Shoah

\section{Histoire sociale}

Résumés / Abstracts

Livres reçus 
Informations éditoriales : http://annales.ehess.fr

Rédaction des Annales

EHESs, 105 boulevard Raspail 75006 Paris (France)

Tél.: +33 (0)153105377

Courriel: annales@ehess.fr

L'édition française des Annales est consultable en accès libre sur les portails suivants:

- Cairn (www.cairn.info/revue-annales.htm), depuis 2001, avec un délai de quatre ans

- Gallica (www.gallica.bnf.fr), 1929 à 1938

- Persée (www.persee.fr/collection/ahess), 1929-1932, 1939-1941, 1943-2002

et sur abonnement sur les portails suivants :

- Cambridge Core (www.cambridge.org/annales), depuis 1929

- Jstor (www.jstor.org/journal/annahistscisoc), depuis 1929, avec un délai de cinq ans

L'édition anglaise des Annales est consultable en texte intégral sur les portails suivants:

- Cairn international (www.cairn-int.info/journal-annales.htm), 2012 à 2016

- Cambridge Core (www.cambridge.org/annales-in-English), depuis 2012

- Revues.org (www.revues.org/annales-english-edition), 2012 à 2016

Depuis 2017, la revue des Annales est publiée en version imprimée et en ligne par les Éditions de l'Ehess en partenariat avec Cambridge University Press. Les abonnements peuvent être achetés auprès de votre agence d'abonnement habituelle ou directement auprès de Cambridge University Press :

Cambridge University Press (Journals)

University Printing House

Shaftesbury Road

Cambridge CB2 8BS (Royaume-Uni)

journals@cambridge.org

Les abonnements débutent au premier numéro de l'année; tarifs et bulletin d'abonnement se trouvent en fin de numéro et à l'adresse www.cambridge.org/annales/subscribe-fr.

Vente au numéro (France, Belgique, Suisse) : $25 €$

Diffusion en librairie

CDE (Centre de diffusion de l'édition), 17 rue de Tournon 75006 Paris (France)

Distribution

Sodis, 128 avenue du Maréchal-de-Lattre-de-Tassigny 77400 Lagny-sur-Marne (France)

Vente au numéro pour le reste du monde: veuillez contacter Cambridge University Press

Maquette : Michel Rohmer

(C) École des hautes études en sciences sociales, Paris, 2018

ISSN (édition française) : 0395-2649 (version imprimée) et 1953-8146 (version numérique) 


\section{Annales}

Histoire, Sciences Sociales

Fondateurs: Marc Bloch et Lucien Febvre Ancien directeur: Fernand Braudel

Revue trimestrielle publiée depuis 1929 , éditée par l'École des hautes études en sciences sociales, avec le concours de la Fondation Florence Gould et de l'American University of Paris

\section{Comité scientifique}

Mary Beard, Jane Burbank, Sandro Carocci, Jocelyne Dakhlia, Lorraine Daston, Marc Ferro, François Hartog, António Manuel Hespanha, Christian Lamouroux, Emmanuel Le Roy Ladurie, Alf Lüdtke, André Orléan, Jacques Poloni-Simard, Jacques Revel, Pierre-François Souyri, Sanjay Subrahmanyam, Laurent Thévenot, Lucette Valensi

\section{Directeur de la rédaction}

Vincent Azoulay

\section{Comité de rédaction}

Étienne Anheim, Romain Bertrand, André Burguière, Guillaume Calafat,

Vanessa Caru, Jean-Yves Grenier, Catherine Rideau-Kikuchi, Camille Lefebvre, Antoine Lilti, Antonella Romano, Anne Simonin, Michael Werner

\section{Responsables de la version anglaise}

Nicolas Barreyre, Stephen W. Sawyer

\section{Secrétariat de la revue}

Aurianne Cox, Livia Foraison, Séverine Guiton, Chloe Morgan, Sophie Muraccioli 
